Case Report

\title{
Post-Streptococcal Syndrome in a 5-Years Old Boy with Sever Vitamin D Deficiency Only Responding to Monoclonal Antibody: First Case Report
}

\author{
Sam Hassan ", Farsheed Zakir, Sameer Sajwani \\ Department of Pediatrics, Mediclinic City Hospital, Dubai, United Arab Emirates \\ Email address: \\ samhassan@gmail.com(S. Hassan), farsheed.zakir@mediclinic.ae(F.Zakir), sameer.sajwani@mediclinic.ae(S. Sajwani) \\ ${ }^{*}$ Corresponding author
}

\section{To cite this article:}

Sam Hassan, Farsheed Zakir, Sameer Sajwani. Post-Streptococcal Syndrome in a 5-Years Old Boy with Sever Vitamin D Deficiency Only Responding to Monoclonal Antibody: First Case Report. American Journal of Pediatrics. Vol. 4, No. 2, 2018, pp. 25-30.

doi: 10.11648/j.ajp.20180402.12

Received: April 23, 2018; Accepted: May 16, 2018; Published: June 12, 2018

\begin{abstract}
Despite the reduction in incidence of Group A Streptococcal (GAS) infections in the last few decades its global burden on mortality and morbidity remained significantly high and represent major concerns in many developing countries. These morbidities and mortalities can be serious in immunocompromised individuals. It has been shown for the last two decades that vitamin D plays an important role in the immune system modulation and its deficiency were associated with recurrent and sever infections. Only few literatures were written about the vitamin D deficiency and GAS infections and very scarce about its complications. One of the rarely reported complications of GAS infections is post-streptococcal systemic vasculitis. The association of this complications and the vitamin D deficiency was not reported before. We report for the first time a 5 years old boy presented with sever post-scarlet fever vasculitis with sever vitamin D deficiency, only responded to monoclonal antibody infusion. The presentation was complicated by more than one complications of GAS infection, hence difficult to diagnose initially and found to have sever vitamin D deficiency which we concluded is the causative factor for these complications. On correction of vitamin D level the deranged immunological and other factors causing the vasculitis were corrected subsequent to vitamin D correction. It is therefore recommended to check vitamin D concentration in cases of sever GAS especially in association with its complications such as the systemic vasculitis.
\end{abstract}

Keywords: Streptococcal Infection, Scarlet Fever, Vitamin D Deficiency, Vasculitis

\section{Introduction}

Group A streptococci (GAS) is a gram positive cocci which is ubiquitous and present with variety of symptoms that may lead to significant morbidity and mortality with global disease burden of more than 18 million cases annually and a death tool estimated more than 500,000 deaths. The infection can be so acute to lead to rapid decline and death especially when the immune system compromised [1]. The prevalence of streptococcal diseases considerably decreased in the industrialized countries but still remains a major public health concern among the developing countries. GAS usually causes 2 type of complications, suppurative and non-suppurative.
The suppurative are more while the rare non-suppurative are due to the effect of the bacterial pyrogen on the affected organ, such as Rheumatic fever, glomerulonephritis, reactive arthritis, Kawasaki disease, pediatric autoimmune neuropsychiatric disorders and the rarely reported systemic vasculitis [2 and 3] The non-suppurative complications arise from certain subtypes of (GAS) which cause an autoimmune response, molecular mimicry [4] Although post-streptococcal vasculitis has been rarely reported but, none of the reported cases presented as Scarlet fever (SF) neither vitamin D level was measured [5].

SF usually followed streptococcal throat infection however in many cases no source of infection. It is usually mild disease and can be self-limiting. It rarely causes Rheumatic fever and 
valvular heart damage. SF usually present with typical lacy like rash sparing the face with tongue and mucus membrane involvement and skin peeling during the recovery phase. Moreover SF may present with silent or subclinical course with only peeling of the skin. It can be difficult to diagnose in the dark skin, hence suspicion based on accompanying symptoms and diagnostic studies are important [6]. It is usually a school age disease oppose to the Kawasaki disease which is preschool. SF incidence increased sharply since 2011 in many countries including the UK, South Korea, Vietnam, Hong Kong and China which triggered an increase in the autoimmune post-streptococcal syndromes [7].

Post streptococcal syndromes usually occur 1-3 weeks after infection. Some syndromes may occur within a few days, making antibody confirmation difficult. Therefore reexamining titers is important. Movement and neuropsychological syndromes may be occult and occur months after the inciting infection. Post streptococcal syndromes are immune mediated and hence factors such as vitamin $\mathrm{D}$ deficiency that derange the immune system may play considerable role in aggravating these complications.

Many literatures and case reports published in the last two decades about the role of vitamin $\mathrm{D}$ in the immune system and its protective role against autoimmune disease and infections. Upper respiratory tract infections and low vitamin D has been suggested for many years. Recent studies have demonstrated the association of respiratory infections and the concentrations of vitamin D [8-11]. Vitamin D plays an important role in the local immune systems such as the production of the antimicrobial peptides (AMPs) in the respiratory tract defensin and cathelcidine [12]. Moreover low level of vitamin D have been shown in cases with autoimmune systemic vasculitis [13]. This case is the first report of association of sever vitamin D deficiency and post SF syndrome including systemic vasculitis that only responded to biological treatment.

\section{Case Report}

A five years old boy with unremarkable past medical history, from French parents, both and the eldest brother are healthy. He was referred from another hospital due to fever, lethargy for 10 days, and later developed scarlentina rash. After admission, he had shortness of breath and found to have large right-side empyema. Exudative fluid $350 \mathrm{ml}$ drained which was negative for bacteria, TB, Cytology, and fungal infection. He was on broad spectrum antibiotics before transfer and started empirical antibiotics of vancomycine, ceftriaxone and later azithromycin. In view of no response and despite the chest US showed full recovery from the empyema the patient continued to spike high fever after 48 hours, clindamycin was added.

The initial investigations showed an evidence of nephritis, deranged liver enzymes, and increased inflammatory markers (Table 1). The child remained unwell with persistent fever and blood tests for rheumatoid diseases, oncology include bone marrow, echocardiography, infections, and renal biopsy were normal. Skin rash started to peel more including the lips (Figure 1) and got mouth ulcers. A diagnosis of atypical Kawasaki disease was considered despite normal echo (only trivial mitral regurgitation) and was given two doses of IVIG $(2 \mathrm{gm} / \mathrm{Kg}$ each time) alongside with Methylprednisolone $(20 \mathrm{mg} / \mathrm{Kg}$ ) for which he had no response and his inflammatory markers remained high. The antibiotics were stopped too.

Table 1. Blood results (arrows indicates repeated results).

\begin{tabular}{l} 
C REACTIVE PROTIEN mg/dL \\
ESR mm/Hr \\
WHITE CELL COUNT K/uL \\
COMPLEMENT C3 g/L \\
COMPLEMENT C4 g/L \\
ALT IU/L \\
AST IU/L \\
Platelets K/Ul \\
ASO TITER IU/mL \\
Hemoglobin g/dL \\
D-DIMER ug/mL FEU \\
FIBRINOGEN mg/dL \\
ANTI-DNase B U/mL \\
\hline
\end{tabular}
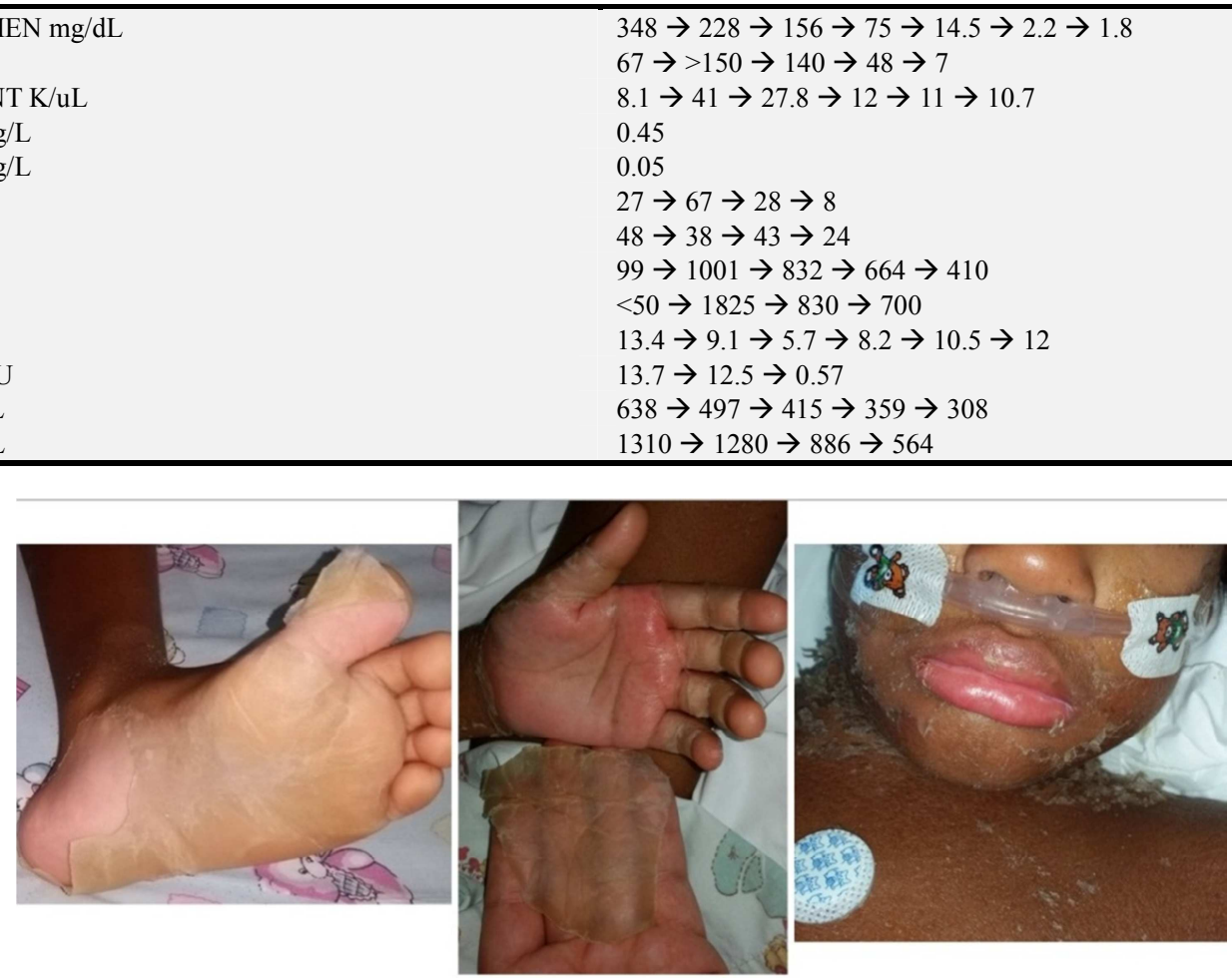

Figures 1. Peeling of the skin. 
The ASOT was less than $50 \mathrm{IU} / \mathrm{ml}$ at the admission and the repeated one after 10 days was $1834 \mathrm{IU} / \mathrm{ml}$ and the anti-Dnase-B strep antibodies $1310 \mathrm{U} / \mathrm{ml}$ (normal ref. values: 0 - 70). Serum Fibrinogen and the D dimer were both increased, suggesting endothelial damage due to systemic vasculitis [2].

After 10 days of empirical antibiotics, no response to IVIG and the results suggesting vasculitis the patients was started on infliximab infusion $(5 \mathrm{mg} / \mathrm{Kg})$ given with Chlorohistol IV, 1 $\mathrm{mg} / \mathrm{Kg}$. The patients then had dramatic improvement clinically, fever subsided for the first time back to normal within few hours of the infusion and FBC, Liver enzymes, urine test, CRP, ESR, fibrinogen, d dimer, and platelets were all improving and normalized after 3 weeks. The patient was on aspirin antiplatelet dose $(5 \mathrm{mg} / \mathrm{Kg} /$ dose OD) which was stopped on follow up and normal echocardiographs.

Before the infliximab the patient $\mathrm{Hb}$ was dropping from 13.4 to 5.7. Blood transfusion was declined by the parents and hence erythropoietin was used SC $(50 \mathrm{U} / \mathrm{Kg}$ three times per week). Erythropoetin was introduced at the time of the infliximab infusion and the $\mathrm{Hb}$ increased to 6 and normalized later on follow up.

It is interestingly to note that the patient got sever vitamin D deficiency $(8.8 \mathrm{ng} / \mathrm{ml})$ which then treated with $10,000 \mathrm{IU}$ orally followed by 2000 IU daily and reduced to 400 IU daily after normalization of blood concentration of vitamin D3. The patient was healthy before and had no other causes than reduced exposure to sun light and dietary to explain his vitamin D deficiency. He has no renal or liver gastrointestinal disorders with normal blood and ultrasound scans of these systems. Interestingly to note here that individual with darker skin got high melanin which acts as a natural sun protection that may reduce the vitamin D production by more than $95 \%$. Hence these people require longer exposure to sun to produce the same amount of vitamin D [14].

The patient was also given IM pencillin $G$ as we were not sure if the mitral regurgitation was due to early carditis in which case he will fit with the diagnosis of Rheumatic fever according to the Jones Criteria. Later on, this diagnosis was refuted by two normal scans and ECG.

During the admission the patient was also investigated for any immunodeficiency disorders, rheumatological disorders, renal disorders, liver diseases, other infections, hematological and tumor diseases and all were normal. These were included FBC, Blood film, bone marrow, renal biopsy, viral screens, HIV screen, Rheumatology screen, immunoglobulins, complement system screen, lymphocytes cell studies, urine protein creatinine ratios, hepatitis screen, coagulation studies, appropriate cultures, and tumor chromosomal studies. The only increased concentrations were are immunoglobulin $\mathrm{G}$ subclasses which will be discussed under discussion.

In summary this 5 years old boy got sever vitamin D deficiency, presented with vague post scarlet fever syndrome including vasculitis, that only responded to infliximab and correction of his vitamin D level.

\section{Discussion}

The patient was initially referred as a case of pyrexia for 10 days, possible lupus nephritis and to exclude leukemia. Bone marrow test was normal and the renal biopsy only showed nonspecific acute tubular necrosis. Patient dark skin makes the diagnosis of the SF clinically challenging especially on the face of normal titers and cultures. Repeated tests showed strong evidence of streptococcal infection (ASOT sero-conversion from less than 50 to $1840 \mathrm{IU} / \mathrm{mL}$ and anti-Dnase B was $1325 \mathrm{U} / \mathrm{mL}$ ). The combined elevation of both revealed a sensitivity of $95 \%$ and specificity of $98 \%$ [15] and with the sero-conversion make the diagnosis even more reliable [16]. The rapid antigen throat swab test for streptococci can be negative and not always reliable in the diagnosis of SF. [17]

The patient vitamin D level was extremely low, the $\mathrm{IgG}$ subclasses 1, 2, and 3 very high (Table 2), had evidence of endothelial injury (vasculitis) and suppurative complication (empyema) of GAS.

Table 2. IgG subclasses; [normal reference values] (arrows show repeated levels).

\begin{tabular}{ll}
\hline Ig G1 & $3276 \rightarrow 2466 \rightarrow 1900 \rightarrow 1367[306-794] \mathrm{mg} / \mathrm{dL}$ \\
Ig G2 & $1038 \rightarrow 529 \rightarrow 376 \rightarrow 215[68-327] \mathrm{mg} / \mathrm{dL}$ \\
Ig G3 & $>213 \rightarrow>213 \rightarrow 212 \rightarrow 119[16-94] \mathrm{mg} / \mathrm{dL}$ \\
Ig G4 & $34 \rightarrow 26 \rightarrow 17 \rightarrow 11[2-96] \mathrm{mg} / \mathrm{dL}$ \\
\hline
\end{tabular}

It is likely that the very low vitamin D might have been for long time paving the way for multiple complications following GAS. The nonresponse to the conventional treatment and the dramatic improvement to the biological treatment signifying the disturbed immune system that resulted from very low vitamin D. He was discharged home 72 hours after infliximab and resolved fully after 3 weeks on follow up.

There are many causes for the vitamin D deficiency. The commonest cause is due to lack of exposure to sun light and dietary. It can happen in strict vegan diet because the main sources for vitamin D are animal based like fish oil, beef, egg yolk and liver. Other causes of vitamin D deficiency are rare and can happen due to renal disorders, liver disorders, and gastrointestinal diseases such as Crohn's disease, ulcerative colitis, cystic fibrosis, coeliac disease. People with high body mass index more than 30 are more susceptible for vitamin D deficiency due to extraction of vitamin $\mathrm{D}$ to the fat cells from the blood. In this patient there was no clinical or biochemical or radiological evidence of secondary causes other than lack of exposure to sun light and possible dietary. The patient's dark skin contributing farther to the lack of vitamin D as that needs more exposure to sun light and high melanin skin acts as a sun screen reducing production up to more than $95 \%$ [14]. The patient responding to therapeutic and then supplement doses of vitamin D orally.

Many studies have demonstrated the increased susceptibility to infection and autoimmune diseases due to vitamin D deficiency [11]. Recent studies have been shown that vitamin D level of less than $30 \mathrm{ng} / \mathrm{ml}$ associated with 
different bacterial and viral infections [18-23]. Vitamin D is an innate and local immune-modulatory regulator which is produced by the immune cells. It inhibits $\mathrm{B}$ and $\mathrm{T}$ cell proliferation so as the immunoglobulin production [24] subsequently decreasing the inflammatory cytokines (IL-17 and 21) and increasing the anti-inflammatory cytokines like IL- 10. It also inhibits monocytes, dendritic cells (DC) and TNF- $\alpha$. Interestingly to note that the patient's monocytes count was high. The DC are important in self-tolerance immune system, in that when the immature DC present the self-antigen it produce tolerance in reverse to the same antigen presented by mature DC. Self-antigen resultant from infection or cell death is abundant in the body. Vitamin D increase self-antigen tolerance by inhibiting maturation of DC. Vasculitis is one of the resultant complications of this autoimmune response to infection [25-26].

In GAS, there are several immune mechanisms may be operable in post streptococcal syndromes such as antibody directed molecular mimicry [27-28] and possible super antigen stimulation of T cells [29-30]. GAS produce pyrogenic exotoxins which is responsible for activation of T-Cells and known to damage the plasma membranes of blood capillaries under the skin hence the red rash of SF. There have been at least 13 types of the pyrogenic exotoxins implicated in different tissue inflammations [31-33]. The commonest are SpeA, SpeB and SpeC. The SpeA \& SpeC bind and activate the T-Cell which then produce pro-inflammatory cytokines and chemokines. This binding happens with low affinity and is nonspecific, hence it allows the superantigens $\mathrm{A}$ and $\mathrm{C}$ to activate many $\mathrm{T}$ Cell reaching up to $50 \%$ of the body T cells. Vitamin D deficiency in this case make more T cells available for the action of the GAS super-antigen. SpeB degrades immunoglobulins and cytokines, as well as through cleavage of $\mathrm{C} 3 \mathrm{~b}$, inhibiting recruitment of phagocytic cells and the complement activation pathway. This results in decreased inflammation and neutrophil levels around the site of infection, preventing clearance and phagocytosis and promoting the survival of GAS pyogenes [34]

The patient's IgG subclasses 1, 2 and 3 were very high. This is likely due to the protracted scarcity of the action of Vitamin $\mathrm{D}$ on the $\mathrm{B}$ cells which ended by escalating the immune-infectious consequences of GAS [24]. The normalization of the IgG subclasses blood levels (table 2) ensued after correction of vitamin D status. The IgG subclasses play an important role in the development of vasculitis by activating the cytokine-primed neutrophils within the vasculature and the complement system leading to rapid intensification of inflammation and recruitment of monocytes causing endothelial damage and consequential vasculitis. It is known that in normal individuals the human IgG antibodies provide multiple protective mechanisms through their multifunctional adapted molecule whereas the $\mathrm{Fab}$ region binds to the antigens and the FC region to the multiple protective effectors ligands. Potential pathogens such as streptococci evolve strategies to frustrate and/or evade these protective mechanisms [35]

Moreover, epidemiological studies have shown the association between the vitamin D deficiency and chest infection, empyema (Rachtic lung) and TB [36-40]

Interestingly the patient presented with chest infection and empyema. It is the extravagant action of GAS due to vitamin D deficiency which lead to the compound complications. Moreover the patient dramatically responded to infliximab. It has been shown in many studies that infliximab is having evolving multiple mechanism pathways to eliminate the complex cytokines and chemokines-dependent inflammatory pathways [41-42] Infliximab may have a role in vasculitis not responding to other conventional treatment such as $\mathrm{KD}$ and post-infectious vasculitis.

\section{Conclusion}

This case report shows that vitamin D deficiency escalate the immune-infectious process of GAS, leading to compound SF complications including systemic vasculitis that responded only to monoclonal antibodies treatment. It is, therefore, recommend that patients presented with sever GAS infections and or post GAS complications to have their vitamin D status checked and corrected.

\section{Acknowledgements}

The authors are grateful to the parents of the child who allowed to publish this case and all professionals and departments involved in the management.

\section{Authors' Contributions}

The case report was prepared mainly by Sam Hassan

Farshed Zakir helped in tables and results

Sameer Sajwan involved in management and reviewing of the manuscript

\section{Disclosure Policy}

The authors declare that there is no conflict of interest regarding the publication of this paper.

\section{References}

[1] Thompson K, Sterkel A K, McBride J A, and Corliss R F; The Shock of Strep: Rapid Deaths Due to Group A Streptococcus; 2018; Acad Forensic Pathol. 8(1): 136-149.

[2] Martins AL, Figueiredo AE, Brito MJ (2015) Vasculitis, a Rare Presentation of a Post-Streptococcal Syndrome. Clin Res Infect Dis 2(1): 1011.

[3] Brahmadathan NK (2017); Molecular biology of Group A Streptococcus and its complications in vaccine strategies; Indian J of Med Microbiol; 35: 176-183.

[4] Rajgor AD, Hakim NA, Ali S and Darr Adnan, (2018); Paediatric Autoimmune Neuropsychatric Disorder Associated with Group A Beta-Haemolytic Streptococcal infection: An indication for tonsillectomy? A review of the literature; Internation $\mathrm{J}$ of Otolaryngology cited on: https://doi.org/10.1155/2018/2681304. 
[5] Martins AL, Figueiredo AE, Brito MJ (2015) Vasculitis, a Rare Presentation of a Post-Streptococcal Syndrome. Clin Res Infect Dis 2(1): 1011 .

[6] Zitelli, Basil; McIntire, Sara; Nowalk, Andrew (2018). Zitelli and Davis' Atlas of Pediatric Physical Diagnosis. Elsevier, Inc.

[7] You Y, Davies M R, Protani M, McIntyre L, Walker M J, and Zhang J; (2018); Scarlet Fever Epidemic in China Caused by Streptococcus pyogenes Serotype M12: Epidemiologic and Molecular Analysis; EBioMedicine 28: 128-135.

[8] Wei L, Xianfen C, Linying G, Hongri L, Cunrong S, Xiaodai C, Qi Z, and Guowei S, (2018); Association between serum 25-hydroxyvitamin D concentration and pulmonary infections in children; Medicine; 97: 1: p e9060; doi: 10.1097/MD.0000000000009060.

[9] Berry DJ, Hesketh K, Power C, Hypponen E. Vitamin D status has a linear association with seasonal infections and lung function in British adults. Br J Nutr 2011; 106:1433-40.

[10] Mulrennan S, Knulman M, Walsh J P, Hue J, Hunter M, Divitini M, Zhu K, Cooke B R, Musk A W, and James A; (2018); Vitamin D and respiratory health in the Busselton Healthy Ageing Study; Respirology (2018). Cited on DOI: $10.1111 /$ resp.13239.

[11] Dąbrowska-Leonik N, Bernatowska E, Pac M, Filipiuk W, Mulawka J, Pietrucha B, Heropolitańska-Pliszka E, Bernat-Sitarz K, Wolska-Kuśnierz B, and Mikołuć B. (2018); Vitamin D deficiency in children with recurrent respiratory infections, with or without immunoglobulin deficiency. Adv Med Sci. 63(1):173-178.

[12] Liu PT, Stenger S, Li H, Wenzel L, Tan BH, Krutzik SR, et al. Toll-like receptor Triggering of a vitamin D-mediated human antimicrobial response. Science 2006; 311:1770-3.).

[13] Holland M et al; Hypogalactosylation of serum $\operatorname{IgG}$ in patients with ANCA-associated systemic vasculitis; Clin Exp Immunol 2002; 129:183-190.

[14] Nair R and Maseeh A; Vitamin D: The sunshine vitamin; 2012; J Pharmacol Pharmacother; 3 (2): 116 - 126.

[15] Blyth CC, Robertson PW. Anti-streptococcal antibodies in the diagnosis of acute and post-streptococcal disease: streptokinase versus streptolysin $\mathrm{O}$ and deoxyribonuclease $\mathrm{B}$. Pathology. 2006;38(2):152-6.

[16] Johnson DR, Kurlan R, Leckman J, Kaplan EL. The Human Immune Response to Streptococcal Extracellular Antigens: Clinical, Diagnostic, and Potential Pathogenetic Implications. Clinical Infectious Diseases. 2010; 50(4):481-490.

[17] Mahyar A, Ayazi P, Safari S, Dalirani R, Javadi A, and Esmaeily S. (2018); Association between vitamin D and urinary tract infection in children; Korean J Pediatr; 61(3): 90 94. doi: 10.3345/kjp.2018.61.3.90.

[18] Bikle D. Nonclassic actions of vitamin D. J Clin Endocrinol Metab. 2009; 94(1):26-34. [PubMed: 18854395].

[19] Ginde AA, Mansbach JM, Camargo CA Jr. Association between serum 25-hydroxyvitamin $\mathrm{D}$ level and upper respiratory tract infection in the Third National Health and Nutrition Examination Survey. Arch Intern Med. 2009; 169(4):384-90. [PubMed: 19237723].

[20] Laaksi I, et al. An association of serum vitamin D concentrations $<40 \mathrm{nmol} / \mathrm{L}$ with acute respiratory tract infection in young Finnish men. Am J Clin Nutr. 2007; 86(3):714-7. [PubMed: 17823437].

[21] Cannell JJ, et al. Epidemic influenza and vitamin D. Epidemiol Infect. 2006; 134(6):1129-40. [PubMed: 16959053].

[22] Bodnar LM, Krohn MA, Simhan HN. Maternal vitamin D deficiency is associated with bacterial vaginosis in the first trimester of pregnancy. J Nutr. 2009; 139(6):1157-61. [PubMed: 19357214].

[23] Amado CA, and Perez MR; (2018); Vitamin D nutritional status and infectious diseases; J Infect Dis Ther 2018, 6:1; 349; DOI: $10.4172 / 2332-0877.1000349$.

[24] Rodriguez M, et al. High frequency of vitamin D deficiency in ambulatory HIV-Positive patients. AIDS Res Hum Retroviruses. 2009; 25(1):9-14. [PubMed: 19108690]).

[25] Lemire JM, et al. 1 alpha, 25-dihydroxyvitamin D3 suppresses proliferation and immunoglobulin production by normal human peripheral blood mononuclear cells. J Clin Invest. 1984; 74(2):657-61. [PubMed: 6611355].

[26] Almerighi C, et al. 1Alpha, 25-dihydroxyvitamin D3 inhibits CD40L-induced pro-inflammatory and immunomodulatory activity in human monocytes. Cytokine. 2009; 45(3):190-7. [PubMed: 19186073]

[27] Cynthia Aranow, Vitamin D and the Immune System; J Investig Med. 2011 August; 59(6): 881-886. doi:10.231/JIM.0b013e31821b8755.

[28] Quinn A, Kosanke S, Fischetti VA, Factor SM, Cunningham MW. Induction of Autoimmune Valvular Heart Disease by Recombinant Streptococcal M Protein. Infect. Immun. 2001; 69(6):4072-4078.

[29] Martins TB, Veasy LG, Hill HR. Antibody responses to group A streptococcal infections in acute rheumatic fever. Pediatr Infect Dis J. 2006; 25(9):832-7. Proft T, Fraser JD. Streptococcal superantigens. Chem Immunol Allergy. 2007; 93:1-23.

[30] Davison SC, Allen MH, Mallon E, Barker JN. Contrasting patterns of streptococcal superantigen-induced T-cell proliferation in guttate vs. chronic plaque psoriasis. $\mathrm{Br} \mathrm{J}$ Dermatol. 2001; 145(2):245-251.

[31] Chen CL, Wu YY, Lin CF, Kuo C-F, Han CL, Wang S, Chuang W-J, Chen C-Y, Wu J-J, Tsai P-J, Liu C-C and Lin YS; (2018); Streptococcal pyrogenic exotoxin B inhibits apoptotic cell clearance by macrophages through protein $\mathrm{S}$ cleavage; Scientific Reports; 6, 26026; doi:10.1038/srep26026.

[32] Knöll H, Srámek J, Vrbová K, Gerlach D, Reichardt W, Köhler W (December 1991). "Scarlet fever and types of erythrogenic toxins produced by the infecting streptococcal strains". Zentralbl Bakteriol. 276 (1): 94-106. doi:10.1016/s0934-8840(11)80223-9. PMID 1789905.).

[33] Brosnahan, A.J.; Schlievert, P.M. (December 2011). "Gram-positive bacterial superantigen outside-in signaling causes toxic shock syndrome: Superantigen outside-in signaling". The FEBS Journal. 278: 4649-67. doi:10.1111/j.1742-4658.2011.08151.x. PMC 3165073 . PMID 21535475).

[34] Holand $\mathrm{M}$ et al; Hypogalactosylation of serum $\operatorname{IgG}$ in patients with ANCA associated systemic vasculitis; Clin Exp Immunol 2002; 129:183-190). 
[35] Najada AS, Habashneh MS, Khader M. The frequency of nutritional rickets among hospitalized infants and its relation to respiratory diseases. J Trop Pediatr; 2004; 50:364-368.

[36] Sabetta JR, DePetrillo P, Cipriani RJ, Smardin J, Burns LA, Landry ML. Serum 25- hydroxyvitamin D and the incidence of acute viral respiratory tract infections in healthy adults. PLoS One 2010; 14:1108.

[37] Berry DJ, Hesketh K, Power C, Hypponen E. Vitamin D status has a linear association with seasonal infections and lung function in British adults. Br J Nutr 2011; 106:1433-40. 17.

[38] Grant WB. Variation in vitamin D production could possibly explain the seasonality of childhood respiratory infections in Hawaii. Pediatr Infect Dis J 2008; 27:853.

[39] Ginde AA, Mansbach JM, Camargo Jr CA. Association between serum 25- hydroxyvitamin D level and upper respiratory tract infection in the Third National Health and Nutrition Examination Survey. Arch Intern Med 2009;169: 384-90.

[40] Aydin S, Aslan I, Yildiz I, Agachan B, Toptas B, Toprak S, et al. Vitamin D levels in children with recurrent tonsillitis. Int $\mathrm{J}$ Pediatr Otorhinolaryngol 2011; 75:364-7.

[41] Eleftheriou D, Melo M, Marks SD, Tullus K, Sills J, Cleary G, DolezalovaSeza P, Clarissa O, Woo PP et al; (2009); Biologic therapy in primary systemic vasculitis of the young; Rheumatology;48:978-986; doi:10.1093/rheumatology/kep148.

[42] Marcelo Derbli Schafranski and Giuliano Doretto Campanari Infliximab for Idiopathic Deep Cutaneous Vasculitis Refractory to Cyclophosphamide; International Journal of Vascular Medicine, Volume 2010 (2010), Article ID 951850, 2 pages http://dx.doi.org/10.1155/2010/951850. 